\title{
ASCORBATE-ENHANCED CHONDROGENESIS OF ATDC5 CELLS
}

\author{
F M Altaf ${ }^{1,2}$, T M Hering ${ }^{1,2}$, N H Kazmi², J U Yoo 2 , B Johnstone ${ }^{1,2}$ \\ Departments of ${ }^{1}$ Anatomy and ${ }^{2}$ Orthopaedics, Case Western Reserve University, Cleveland, OH 44106, USA.
}

\begin{abstract}
The ATDC5 cell line exhibits the multistep chondrogenic differentiation observed during endochondral bone formation. However, it takes up to two months to complete the process of cell expansion, insulin addition to promote differentiation and further changes in culture conditions effectively to induce hypertrophy. We sought to produce consistent chondrogenesis with significant hypertrophic differentiation with simpler conditions in a more practical time period. By adding ascorbate, the prechondrogenic proliferation phase was shortened from 21 to 7 days, with production of cartilaginous nodules during the chondrogenic phase, after insulin addition, that were greater in number and larger in size. Immunohistochemistry indicated much greater matrix elaboration and the mRNA expression of sox9, aggrecan and collagen type II were all significantly increased earlier and to a much higher degree when compared with controls. Moreover, there was a robust induction of hypertrophy: Col10a1, Runx2 and Mmp13 were all induced within 7-10 days. In conclusion, addition of ascorbate to ATDC5 cultures shortened the prechondrogenic proliferation phase, produced earlier chondrogenic differentiation, heightened gene expression and robust hypertrophic differentiation, abrogating the need for extended culture times and the changes in culture conditions. This simple modification considerably enhances the practicality of this cell line for studies of chondrogenesis.
\end{abstract}

Key words: chondrogenesis, ATDC5 cells, ascorbate, differentiation, hypertrophy.

\author{
Address for correspondence \\ Brian Johnstone, $\mathrm{PhD}$ \\ Department of Orthopaedics and Rehabilitation, OP31 \\ Oregon Health and Science University \\ Portland \\ OR 97239-3098 \\ USA
}

Phone: (503) 494-9505

Fax: (503) 494-5050

Email: johnstob@ohsu.edu

\section{Introduction}

The bones of the vertebral column, pelvis, and upper and lower limbs, are derived during vertebrate embryogenesis from an initial cartilaginous model. This process, which is referred to as "endochondral ossification", involves a precise series of events including aggregation and differentiation of mesenchymal cells, and proliferation, hypertrophy and death of chondrocytes (DeLise et al., 2000). Modifications in cell proliferation, cell morphology, nature and the amount of extracellular matrix macromolecule production, such as collagens and proteoglycans, characterize each stage of chondrocyte differentiation. Proliferating and maturing chondrocytes mainly synthesize collagen type II, IX, and XI and aggrecan, while hypertrophic chondrocyte synthesize type $\mathrm{X}$ collagen before mineralization of the extracellular matrix.

An excellent in vitro model that exhibits the multistep chondrogenic differentiation observed during endochondral bone formation is the mouse embryonal carcinoma-derived cell line ATDC5 (Atsumi et al., 1990; Shukunami et al., 1997). In culture, with $5 \%$ fetal bovine serum (FBS) and $10 \mu \mathrm{g} / \mathrm{ml}$ insulin present, ATDC5 cells differentiate into type II collagen-expressing chondrocytes, via the cellular condensation stage, to form cartilage nodules (Shukunami et al., 1996). The process of differentiation is started with 3 weeks of expansion culture in medium with $5 \%$ fetal bovine serum (FBS), transferrin and selenium, followed by the addition of insulin to promote the differentiation into type II collagenexpressing chondrocytes, via the cellular condensation stage, with formation of the cartilaginous nodules over the next 21 days. To induce hypertrophy and mineral deposition, the medium is changed from DMEM to $\alpha$ MEM, the $\mathrm{CO}_{2}$ level is switched from $5 \%$ to $3 \%$, and cells are incubated in this condition for a further 21 days (Shukunami et al., 1997). After the growth and expansion of the nodules, hypertrophic chondrocytes appear in association with type $\mathrm{X}$ collagen gene expression and elevation of alkaline phosphatase activity, followed by matrix mineralization (Shukunami et al., 1997; Shukunami et al., 1996). Thus, in the presence of insulin without any additional growth/differentiation factors, ATDC5 cells differentiate sequentially to give rise to condensing prechondrocytes, type II collagen-expressing chondrocytes, type $\mathrm{X}$ collagen-expressing hypertrophic chondrocytes and mineralizing chondrocytes (Shukunami et al., 1996).

To date, one hundred and twenty nine published studies used the ATDC5 cell line as an in vitro model of chondrogenesis, according to the National Center for Biotechnology Information website (http:// www.ncbi.nlm.nih.gov/). For most studies that examined hypertrophy, the conditions outlined above (Shukunami 
et al., 1996) were used. We sought to simplify these conditions to produce a reproducible chondrogenesis with significant hypertrophic differentiation in a more practical time period. Part of the hypertrophic medium used originally included $\alpha$-MEM (Shukunami et al., 1997). This medium contains ascorbate, whereas DMEM does not. Therefore, we experimented with ascorbate supplementation as a means of producing a reliable system of complete chondrogenesis. This was done previously, but only as a means of substituting ascorbate for the change of DMEM/F12 to $\alpha$-MEM; the $\mathrm{CO}_{2}$ level was still switched from $5 \%$ to $3 \%$, and cells were still incubated for a further 21 days (Gori and Demay, 2004). In our studies, we sought conditions that would produce expression of early to late chondrogenic markers that would enhance the practicality of using this cell line for studies of chondrogenesis.

\section{Materials and Methods}

\section{Cell culture}

In control ATDC5 cultures, cells were plated at $1.2 \times 10^{5}$ cells/35 mm well in a 1:1 mixture of DMEM and Ham's F-12 (Cellgro ${ }^{\circledR}$, Mediatech,Inc., Herndon, VA, USA) containing $5 \%$ FBS $\left(\right.$ GIBCO $^{\circledR}$, Grand Island, NY, USA), $10 \mu \mathrm{g} / \mathrm{ml}$ human transferrin (Boehringer Mannheim, Mannheim, Germany), and $3 \times 10^{-8} \mathrm{M}$ sodium selenite (Sigma Chemical, St Louis, MO, USA) as previously described (Shukunami et al., 1997). In the experimental ATDC5 cultures, ascorbate 2-phosphate $(37.5 \mu \mathrm{g} / \mathrm{ml})$ (Wako Chemicals USA, Inc., Richmond, VA, USA) was added to this medium. The cells were grown for 7 days after which the medium was supplemented with $10 \mu \mathrm{g} / \mathrm{ml}$ bovine insulin (BD Biosciences, Bedford, MA, USA) to induce chondrogenesis over the following 21 days. Cells were maintained at $37{ }^{\circ} \mathrm{C}$ in a humidified atmosphere of $5 \% \mathrm{CO}_{2}$ in air for the entire culture period. The medium was replaced every other day. The total cell culture time was 28 days.

\section{Alcian Blue staining}

ATDC5 cells were stained with Alcian Blue at various time points to compare the morphological changes between the control ATDC 5 cells and ascorbate-cultured ATDC5 cells. The cells were washed with PBS, fixed with methanol at $20^{\circ} \mathrm{C}$ for 2 minutes, stained for $0.1 \%$ Alcian blue (Waldeck $\mathrm{GmbH} \&$ Co. KG., Munster, Germany) in $0.1 \mathrm{~N} \mathrm{HCl}$ overnight, rinsed with distilled water, and examined with light microscopy.

\section{Immunohistochemistry}

ATDC5 cells were cultured on glass chamber slides as described above and fixed with methanol-acetone (1:1) at room temperature for 10 minutes at each time point. Cells were then rinsed with PBS three times for five minute each time and the fixed sections were blocked with 5\% BSA for 30 minutes then rinsed again three times with PBS. Sections were incubated with primary antibodies for one hour, and washed with PBS three times: anti-collagen type II (II-II6B3, from the Hybridoma Bank, maintained at the University of Iowa) (1:200 dilution) and anti-type X antibody (kindly provided by Dr. Gary Gibson, Henry Ford Hospital, 1:200 dilution) were used. FITC-linked anti IgG secondary antibodies (Molecular Probes, Eugene, OR, USA) diluted 1:2000 in 5\% BSA in PBS were then added to the sections for 45 minutes, followed by rinsing the sections with PBS three times. Sections were mounted in ProLong Gold antifade reagent with 4',6-diamidino-2phenylindole (DAPI) (Molecular Probes) and examined with fluorescence microscopy (Leica Microsystems, Heidelberg, Germany).

\section{RNA extraction, conventional and quantitative RT- PCR}

Total RNA was extracted from ATDC5 cells with the Nucleospin RNA II kit (BD Biosciences), yielding 15-20 $\mu \mathrm{g}$ total RNA/well. cDNA was then synthesized with the I Script ${ }^{\mathrm{TM}}$ cDNA synthesis kit (Bio-Rad Laboratories, Hercules, CA, USA) that includes a blend of oligo(dt) and random hexamer primers in the reaction mix. For realtime quantitative PCR, the $\mathrm{iQ}^{\mathrm{TM}} \mathrm{SYBR}$ Green Supermix kit (Bio-Rad Laboratories) with the MyiQ ${ }^{\mathrm{TM}}$ single color Real-Time PCR Detection System (Bio-Rad Laboratories) were used. Conventional RT-PCR was done with the PCR Master kit (Roche Diagnostics Corporation, Indianapolis, IN, USA) and a RoboCycler ${ }^{\circledR}$ gradient40 RT-PCR machine( Stratagene ${ }^{\circledR}$, La Jolla, CA, USA). PCR primers for Col2a1: 5'-TTGAGACAGCACGACGTGGAG-3' forward and 5'AGCCAGGTTGC-CATCGCCATA-3' reverse, for Agc1: 5'-AGGACCTGGTAGTGCGAGTG3 ' forward and 5'-GCGTGTGGCGAAGAA-3' reverse, and for Sox9 5'ATCGGTGAACTGAGCAGCGAC-3' forward and 5'-GCCTGCTGCT-TCGACATCCA-3' reverse, were designed with mouse sequences using Oligo 6.8 software (Molecular Biology Insight, Inc., Cascade, CO, USA). Col10a1, Runx2, and Mmp13 primer sequences were obtained from the literature (Enomoto et al., 2000; Martinez et al., 2001; Wang et al., 2001). All PCR products were cloned and sequenced to confirm specificity before proceeding with quantitative assays. The 'standard curve quantitation' method was used for this analysis. For each gene product, a standard curve of plasmid DNA containing cloned PCR products generated using the analysis primers was constructed. All reactions were repeated in triplicate and gene expression levels were normalized to $18 \mathrm{~S}$ rRNA determined for the same sample in the same experiment. When determining the quantitative real-time PCR results, the fold expression increases for both the control and ascorbate-cultured ATDC5 cells were compared with the levels on the first day of culture.

\section{Results and Discussion}

ATDC5 cells have been used extensively to investigate the biochemical and developmental mechanisms occurring throughout the process of chondrogenesis (Akiyama et al., 1999a; Akiyama et al., 1999b; Atsumi et al., 1990; Ito et al., 1999; Shukunami et al., 1997). More than one hundred and twenty studies have been done with ATDC5 cells used as an in vitro chondrogenic model (http:// 


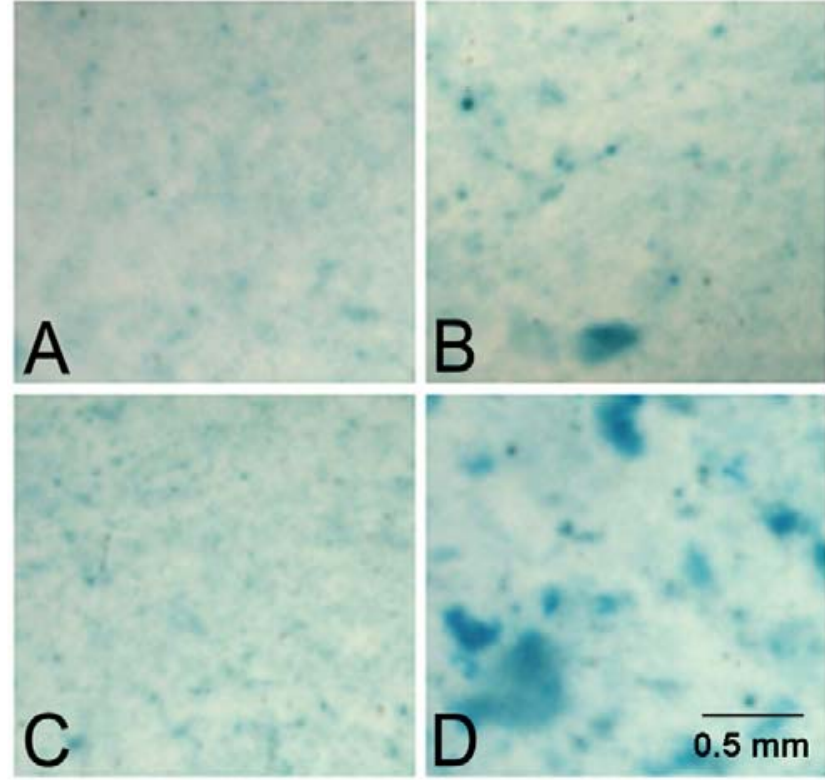

Figure 1: Chondrogenesis was enhanced with ascorbate addition. ATDC5 cells were stained with alcian blue (A-D) at day 7 (A,B) and day 14 (C,D) of chondrogenic culture (post-insulin addition), without $(A, C)$ or with $(B, D)$ ascorbate. Frozen sections of ATDC5 cultures were immunohistochemically stained (E-H) with anti-collagen type II (E,F) and anti-collagen type $\mathrm{X}(\mathrm{G}, \mathrm{H})$ antibodies at day 14 of chondrogenic culture (post-insulin addition). Cells were cultured with $(\mathrm{E}, \mathrm{G})$ or without $(\mathrm{F}, \mathrm{H})$ ascorbate (cell nuclei stained with DAPI, 32x magnification).
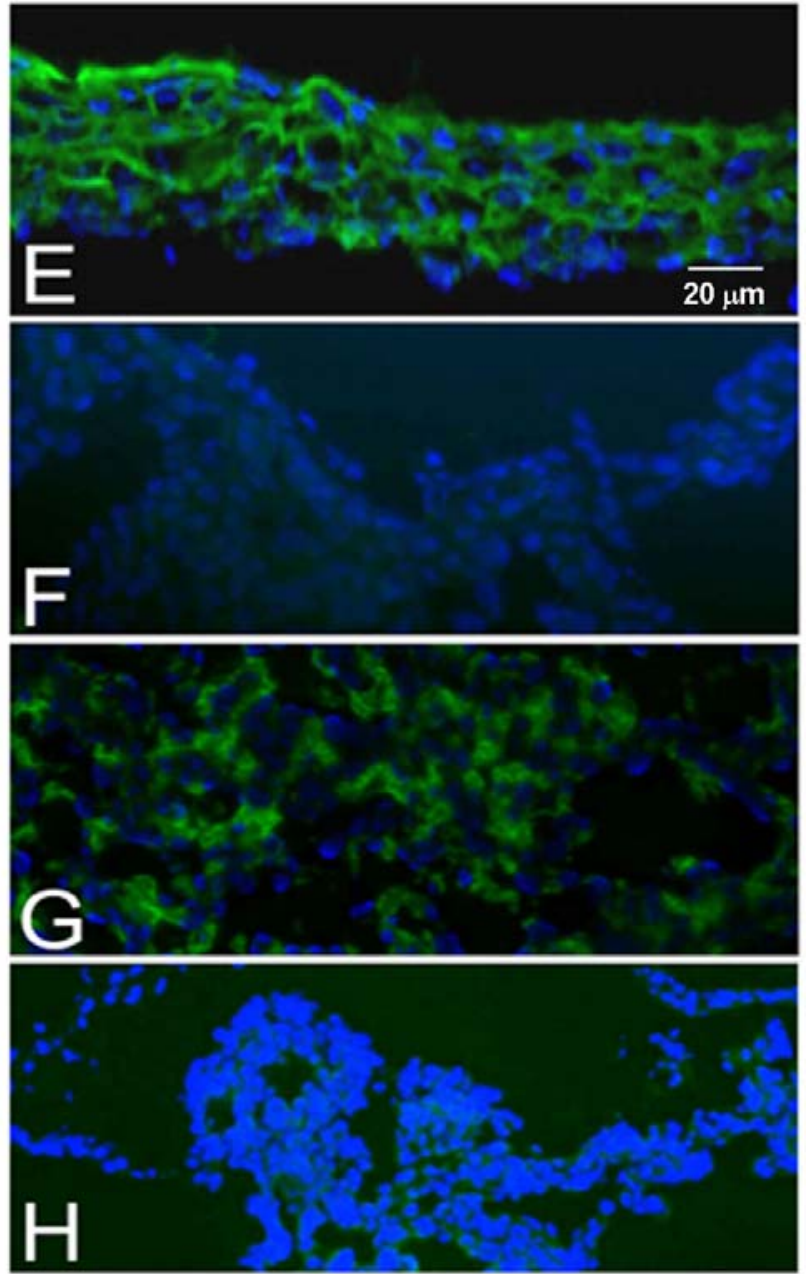

www.ncbi.nlm.nih.gov/). However, to investigate all phases of chondrogenesis, investigators have needed to grow ATDC5 cells in three different media, for extended times up to 63 days, with switches in $\mathrm{CO}_{2}$ levels (Shukunami et al., 1997; Shukunami et al., 1996), and this is still the most common method for working with these cells to induce hypertrophy (Chen et al., 2006; Osawa et al., 2006). The conditions for hypertrophy in ATDC5 cells include switching the DMEM/F12 medium to $\alpha$ MEM, which contains ascorbate. We chose to assess the effect of adding ascorbate from the beginning of the chondrogenic ATDC5 cells culture, seeking a more concise system for enhancing the chondrogenic and hypertrophic differentiation. In previous studies it has been shown that ascorbate regulates the collagen polypeptide synthesis in human skin fibroblasts (Murad et al., 1981) and increases the mRNA expression levels of chondrogenic markers including collagen type II and aggrecan in bovine articular cartilage (Hering et al., 1994). In addition, it was indicated that ascorbate causes an elevation in alkaline phosphatase activity and collagen type $X$ expression in chick chondrocytes (Farquharson et al., 1995; Habuchi et al., 1985; Sullivan et al., 1994). The capacity of ascorbate to stimulate early and late chondrocyte differentiation is related to its requirement as a cofactor for prolyl and lysyl hydroxylases in the synthesis and secretion of stable triple helical collagen (Franceschi, 1992; Robins, 1988). It was also found that ascorbate stimulates glycosaminoglycan (GAGs) synthesis in cultured human skin fibroblasts (Kao et al., 1990).

In the present study, cultures supplemented with ascorbate produced cartilaginous nodules that were greater in number and larger in size (Figure 1A-D). They were also more intensely stained with Alcian Blue, suggesting greater matrix elaboration. In addition, immunohistochemistry indicated that collagen type II and collagen type $\mathrm{X}$ were much more abundant in the extracellular matrix of ATDC5 cells grown with ascorbate when compared with the control ATDC5 cultures (Figure $1 \mathrm{E}-\mathrm{F})$. Collagen type $\mathrm{X}$ was barely detectable in the control cultures. The gene expression of all the chondrogenic markers was increased in cultures containing ascorbate compared with controls (Figure 2). Sox9 expression increased to a small degree in the control cultures between days 4-10. In contrast, it was increased 10 -fold over that of control by day 4 of differentiation culture when ascorbate was present in the medium, and was already 2fold increased on the day of insulin addition (day 0), indicating that the presence of ascorbate accelerates the differentiation of the ATDC5 cells. Concomitant with this, the increases in Col2a1 and Agc1 mRNA also occurred earlier. The Col2a1 gene expression was increased up to 300 -fold and that of $\mathrm{Agc1}$ by up to 150 -fold during the course of the culture, and their highest level of expression 
A

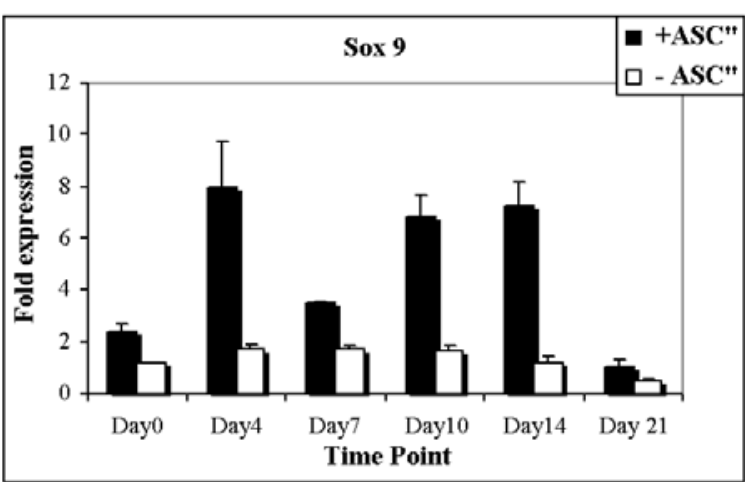

B

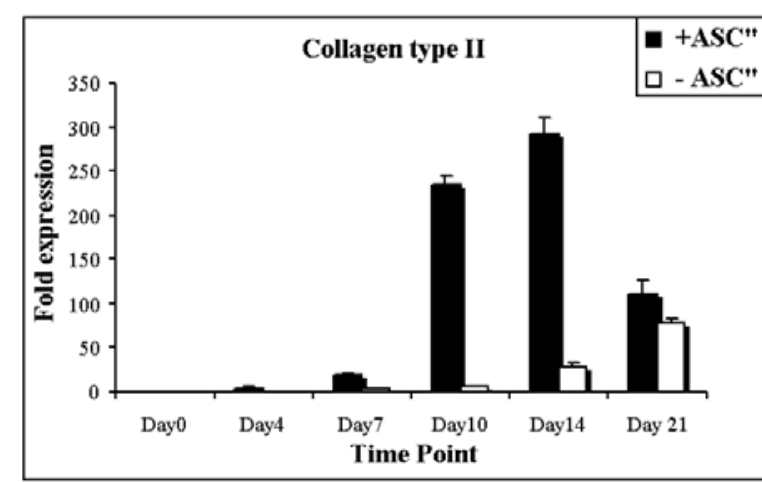

C

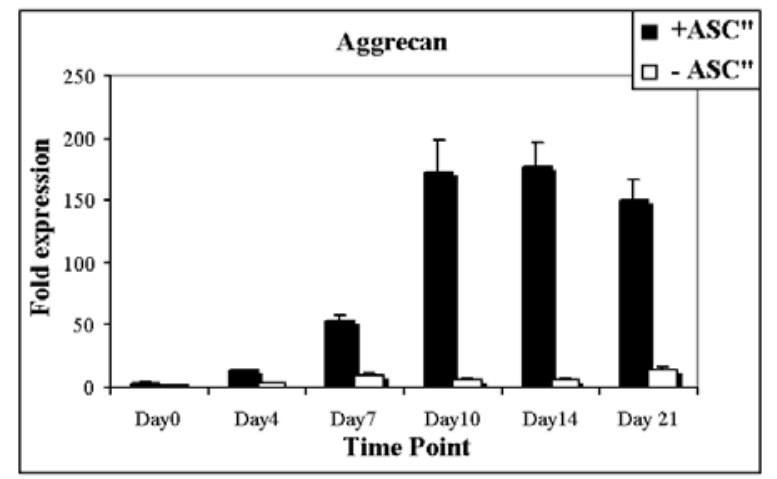

Figure 2: Markers of chondrogenic differentiation were upregulated with ascorbate addition. Quantitative realtime PCR results for mRNA expression levels of sox9 (a), Col2a1 (b) and Agc1 (c). Day 0 is the first day of insulin addition. Three separate cell culture experiments were run, each with $n=3$ replicate measurements for each time points sampled. Means and standard deviations of the three experiments are represented.

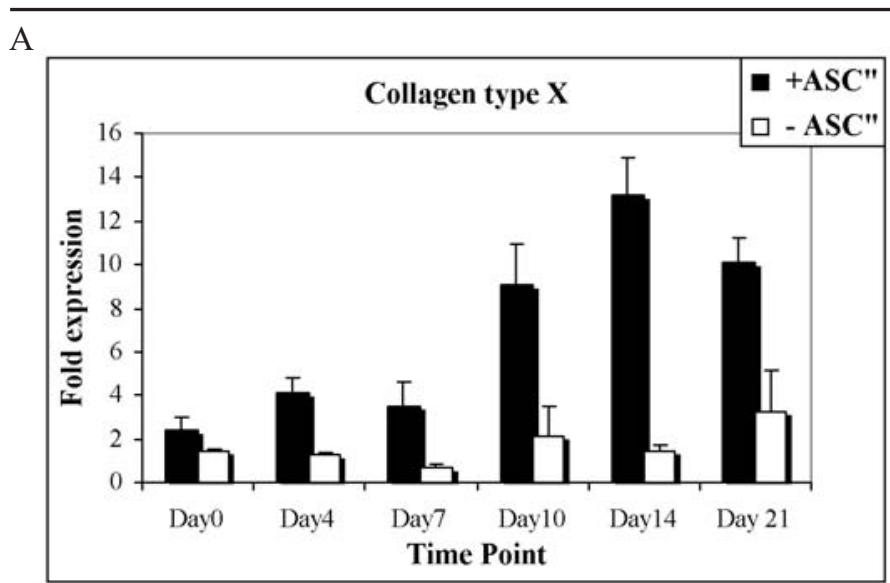

B

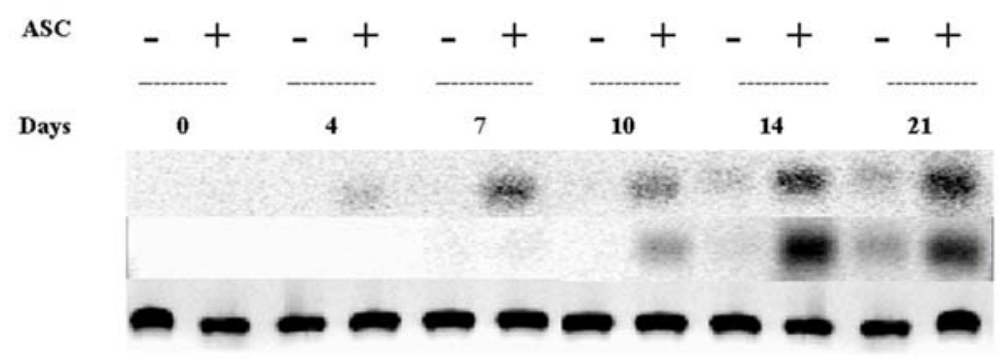

Runx2

MMP13

18s RNA
Figure 3: Markers of chondrocyte hypertrophy were upregulated by ascorbate addition. Quantitative real-time RT-PCR results for mRNA expression levels of Col10a1 and conventional RT-PCR results for mRNA expression of Runx2 and Mmp13. Day 0 is the first day of insulin addition, $(+)$ corresponds to ascorbate treatment, (-) corresponds to non-treated cultures. 
appeared to be earlier by at least 7 days when compared with controls. The level of Col10a1 mRNA expression in control cultures was upregulated approximately 3-fold (Figure 3A), indicating minimal hypertrophic differentiation occurred in the cells, in agreement with the immunohistochemistry results (Figure $1 \mathrm{G}, \mathrm{H}$ ). However, the expression of Col10a1 was increased 14-fold in ascorbate-treated cultures (Figure 3A) and was clearly detectable at the protein level by immunohistochemistry (Figure 1G).

Increased mRNA expression of chondrogenic markers in response to ascorbate was reported in previous work with bovine articular cartilage chondrocytes (Hering et al., 1994). The increased mRNA levels of chondrogenic markers in the present study correlated with the elaboration of a more extensive extracellular matrix in ascorbate-added conditions: immunohistochemistry with anti-collagen antibodies produced more intense staining for collagen types II and X in ascorbate-added cultures when compared with controls. Ascorbate exerts this matrix-inductive effect with transcriptional regulation of the alpha subunit of prolyl 4-hydroxylase and collagens types II and X, as well as post-translational regulation of collagen hydroxylation (Sullivan et al., 1994). The effect on sox9 gene expression has not been previously reported, but the effect on Aggrecan gene expression has been previously documented in bovine chondrocytes, although the mechanism was not determined (Hering et al., 1994). Moreover, the highest level of expression for the chondrogenesis marker genes was earlier in the ascorbatetreated cultures.

The level of hypertrophic differentiation of ATDC5 cells is very low if ascorbate is not added, consistent with previous work that involved the switch in culture conditions (Shukunami et al., 1997), and more recent studies indicating only a 2 to 3 -fold increase of Col10a1 expression in 24 days of culture with insulin by quantitative RT-PCR (Wang et al., 2004). In contrast, addition of ascorbate promoted significant Col10a1 mRNA expression, which correlates with results of previous studies in which ascorbate has been shown to enhance hypertrophic differentiation in other cell lines and primary chondrocytes (Farquharson et al., 1995; Habuchi et al., 1985; Sullivan et al., 1994). However, detection of increased expression of collagen type $\mathrm{X}$ is not sufficient to assess hypertrophy. To confirm the hypertrophic differentiation of the cells we also examined the expression patterns of other hypertrophic markers, Runx2 (Cbfa1) and matrix metalloproteinase-13 (Mmp13, collagenase 3), by conventional RT-PCR (Figure 3B). Runx2 is a member of the runt family of transcription factors that, besides its role in osteogenesis, is required for hypertrophic chondrocyte differentiation. In Runx2-deficient mice hypertrophic chondrocytes are absent from several, although not all, skeletal elements (Inada et al., 1999). It is expressed in prehypertrophic chondrocytes and its constitutive expression induces hypertrophic chondrocyte differentiation, Ihh expression, and endochondral bone formation (Takeda et al., 2001). The expression of Runx2, has been previously reported to be upregulated in prehypertrophic and hypertrophic ATDC5 cells (Enomoto et al., 2000). Runx2 was found to be expressed in ascorbatetreated ATDC5 cell cultures beginning at days 7, whereas it was undetectable before day 14 in non treated ATDC5 cell cultures. The expression of Mmp13 was also detected earlier in ascorbate treated ATDC5 cell cultures (day 7-10 versus day 21 in non-treated cultures) and was upregulated to a higher degree than in controls. Mmp13 plays an important role in bone formation and remodeling and is expressed in both terminal hypertrophic chondrocytes in the growth plate and in osteoblasts (Stickens et al., 2004). The expression of Mmp13 is restricted to the very last rows of hypertrophic chondrocytes and the osteoblasts of the trabecular bone (Stickens et al., 2004). The expression pattern of these markers and that of Col10a1 are consistent with ascorbate's previously documented role in hypertrophy (Farquharson et al., 1995; Habuchi et al., 1985; Sullivan et al., 1994). The mechanism for this is still incompletely understood but it has been documented that ascorbate enhances 1,25-dihydroxyvitamin D3 synthesis, accompanied by upregulation of the vitamin D receptor, suggesting that it may be causing amplification of the vitamin D receptor-dependent genomic response to 1,25-dihydroxyvitamin $\mathrm{D}$, resulting in promotion of terminal differentiation (Farquharson et al., 1998).

In conclusion, addition of ascorbate to ATDC5 cell culture allows the proliferative phase to be reduced to seven days and the chondrogenic phase, including hypertrophy detectable at the gene and protein level, to be considerably shortened compared with the previous method (Shukunami et al., 1997). Addition of ascorbate at the beginning of culture eliminated the need for the switch in the $\mathrm{CO}_{2}$ levels and the culture medium later in the culture. Addition of ascorbate both accelerated and increased the expression of early to late chondrogenic markers. This simple change in the conditions produces a system that considerably enhances the usefulness of the ATDC5 cell as a chondrogenic cell line.

\section{References}

Akiyama H, Kanno T, Ito H, Terry A, Neil J, Ito Y, Nakamura T (1999a) Positive and negative regulation of chondrogenesis by splice variants of PEBP2alphaA/ CBFalpha1 in clonal mouse EC cells, ATDC5. J Cell Physiol 181:169-178.

Akiyama H, Shigeno C, Iyama K, Ito H, Hiraki Y, Konishi J, Nakamura T (1999b) Indian hedgehog in the late-phase differentiation in mouse chondrogenic EC cells, ATDC5: upregulation of type $X$ collagen and osteoprotegerin ligand mRNAs. Biochem Biophys Res Commun 257:814-820.

Atsumi T, Miwa Y, Kimata K, Ikawa Y (1990) A chondrogenic cell line derived from a differentiating culture of AT805 teratocarcinoma cells. Cell Differ Dev 30:109-116.

Chen L, Fink T, Ebbesen P, Zachar V (2006) Temporal transcriptome of mouse ATDC5 chondroprogenitors differentiating under hypoxic conditions. Exp Cell Res 312:1727-1744. 
DeLise AM, Fischer L, Tuan RS (2000) Cellular interactions and signaling in cartilage development. Osteoarthritis Cartilage 8:309-334.

Enomoto H, Enomoto-Iwamoto M, Iwamoto M, Nomura S, Himeno M, Kitamura Y, Kishimoto T, Komori $\mathrm{T}$ (2000) Cbfal is a positive regulatory factor in chondrocyte maturation. J Biol Chem 275:8695-8702.

Farquharson C, Berry JL, Mawer EB, Seawright E, Whitehead CC (1998) Ascorbic acid-induced chondrocyte terminal differentiation: the role of the extracellular matrix and 1,25-dihydroxyvitamin D. Eur J Cell Biol 76:110-118.

Farquharson C, Berry JL, Mawer EB, Seawright E, Whitehead CC (1995) Regulators of chondrocyte differentiation in tibial dyschondroplasia: an in vivo and in vitro study. Bone 17:279-286.

Franceschi RT (1992) The role of ascorbic acid in mesenchymal differentiation. Nutr Rev 50:65-70.

Gori F, Demay MB (2004) BIG-3, a novel WD-40 repeat protein, is expressed in the developing growth plate and accelerates chondrocyte differentiation in vitro. Endocrinology 145:1050-1054.

Habuchi H, Conrad HE, Glaser JH (1985) Coordinate regulation of collagen and alkaline phosphatase levels in chick embryo chondrocytes. J Biol Chem 260:1302913034.

Hering TM, Kollar J, Huynh TD, Varelas JB, Sandell LJ (1994) Modulation of extracellular matrix gene expression in bovine high-density chondrocyte cultures by ascorbic acid and enzymatic resuspension. Arch Biochem Biophys 314:90-98.

Inada M, Yasui T, Nomura S, Miyake S, Deguchi K, Himeno M, Sato M, Yamagiwa H, Kimura T, Yasui N, Ochi T, Endo N, Kitamura Y, Kishimoto T, Komori T (1999) Maturational disturbance of chondrocytes in Cbfa1deficient mice. Dev Dyn 214:279-290.

Ito H, Akiyama H, Shigeno C, Nakamura T (1999) Noggin and bone morphogenetic protein-4 coordinately regulate the progression of chondrogenic differentiation in mouse clonal EC cells, ATDC5. Biochem Biophys Res Commun 260:240-244.

Kao J, Huey G, Kao R, Stern R (1990) Ascorbic acid stimulates production of glycosaminoglycans in cultured fibroblasts. Exp Mol Pathol 53:1-10.

Martinez ME, Sampliner R, Marshall JR, Bhattacharyya AK, Reid ME, Alberts DS (2001) Adenoma characteristics as risk factors for recurrence of advanced adenomas. Gastroenterology 120:1077-1083.

Murad S, Grove D, Lindberg KA, Reynolds G, Sivarajah A, Pinnell SR (1981) Regulation of collagen synthesis by ascorbic acid. Proc Natl Acad Sci U S A 78:2879-2882.

Osawa A, Kato M, Matsumoto E, Iwase K, Sugimoto T, Matsui T, Ishikura H, Sugano S, Kurosawa H, Takiguchi M, Seki N (2006) Activation of genes for growth factor and cytokine pathways late in chondrogenic differentiation of ATDC5 cells. Genomics 88:52-64.

Robins SP (1988) Functional properties of collagen and elastin. Baillieres Clin Rheumatol 2:1-36.

Shukunami C, Ishizeki K, Atsumi T, Ohta Y, Suzuki F, Hiraki Y (1997) Cellular hypertrophy and calcification of embryonal carcinoma-derived chondrogenic cell line ATDC5 in vitro. J Bone Miner Res 12:1174-1188.

Shukunami C, Shigeno C, Atsumi T, Ishizeki K, Suzuki F, Hiraki Y (1996) Chondrogenic differentiation of clonal mouse embryonic cell line ATDC5 in vitro: differentiationdependent gene expression of parathyroid hormone (PTH)/ PTH-related peptide receptor. J Cell Biol 133:457-468.

Stickens D, Behonick DJ, Ortega N, Heyer B, Hartenstein B, Yu Y, Fosang AJ, Schorpp-Kistner M, Angel P, Werb Z (2004) Altered endochondral bone development in matrix metalloproteinase 13-deficient mice. Development 131:5883-5895.

Sullivan TA, Uschmann B, Hough R, Leboy PS (1994) Ascorbate modulation of chondrocyte gene expression is independent of its role in collagen secretion. J Biol Chem 269:22500-22506.

Takeda S, Bonnamy JP, Owen MJ, Ducy P, Karsenty G (2001) Continuous expression of Cbfa 1 in nonhypertrophic chondrocytes uncovers its ability to induce hypertrophic chondrocyte differentiation and partially rescues Cbfa1-deficient mice. Genes Dev 15:467481.

Wang D, Canaff L, Davidson D, Corluka A, Liu H, Hendy GN, Henderson JE (2001) Alterations in the sensing and transport of phosphate and calcium by differentiating chondrocytes. J Biol Chem 276:33995-34005.

Wang G, Woods A, Sabari S, Pagnotta L, Stanton LA, Beier F (2004) RhoA/ROCK signaling suppresses hypertrophic chondrocyte differentiation. J Biol Chem 279:13205-13214.

\section{Discussion with Reviewers}

F. Barry: How relevant is the use of ATDC5 cells compared to primary mesenchymal progenitor cells in this particular area of research?

Authors: In comparison with primary mesenchymal progenitor cells, ATDC5 cells are advantageous because they are consistent in their chondrogenic gene expression profiles whenever cultured. Moreover, they are easier to transfect and readily available.

F. Barry: Has the use of these cells been superseded by newly available human embryonic stem cell lines?

Authors: The consistent differentiation process, the availability, and the convenience favour the use of ATDC5 cells over embryonic stem cell lines, at least for the present time. They also are free of the emotive issues surrounding human embryonic cell lines.

F. Barry: Why has it taken so long to recognise the importance of ascorbate in this system, since the biochemical role of ascorbate has been described for several decades?

Authors: Although the biochemical roles of ascorbate in collagen synthesis and chondrocyte metabolism have been described for several decades, its effect on ATDC5 cell culture and hypertrophy was not tested. We can only speculate as to why this was. Since many researchers only 
look for gene level responses to perturbations in ATDC5 cells, they have not paid attention to the extracellular protein production and thus may not have considered the use of ascorbate.

C. Hartmann: Surprisingly, in contrast to what has been observed during in vivo cartilage development, the transcriptional expression of prehypertrophic markers such as Runx 2 and the marker for hypertrophic chondrocytes such as Col10al preceded the expression of Col2a1, a marker for more immature chondrocytes, in ATDC 5 cells. Is this true for other prehypertrophic markers, such as Ihh as well? What could be possible explanations?

Authors: The increase in Col2a1 expression is almost 300 -fold by day 10 (figure 2B). When this is graphed, the earliest small changes are not seen. The expression for Col2al at day zero (first day of insulin addition) is almost 2-fold increased over the cells at day zero of plating into maintenance medium (one week prior to insulin addition). The Col10a1 expression is 14 fold higher at its maximum, making earlier day values more visible. That stated, it is also about 2-fold increased from the culture initiation day. This is not without precedent. In recent work by (Mwale et al., 2006), it was shown that Col10a1 is expressed early during the chondrogenic differentiation in bone marrow derived mesenchymal cells. We detect early Col10a1 gene expression by quantitative PCR, but cannot detect the protein until later in culture, in contrast to Col2a1, but this may be a sensitivity issue.

Although Runx2 is known to be activated in prehypertrophic chondrocytes, it is also expressed early in chondrogenic mesenchymal cells, and then its expression declines in the early differentiated chondrocyte (Lefebvre and Smits, 2005). This may be the reason for the detection of early gene expression of Runx 2 in ATDC5 cell cultures.

It must be emphasized that culture systems such the one used here are not synchronized and cells may be undergoing differentiation with different timing. Furthermore, not every cell in the culture undergoes the differentiation, as evidenced by the nodular pattern.

\section{Additional References}

Lefebvre V, Smits P (2005) Transcriptional control of chondrocyte fate and differentiation. Birth Defects Res C Embryo Today 75: 200-212.

Mwale F, Stachura D, Roughley P, Antoniou J (2006) Limitations of using aggrecan and type $\mathrm{X}$ collagen as markers of chondrogenesis in mesenchymal stem cell differentiation. J Orthop Res 24: 1791-1798. 